\title{
Quantum Energy Teleportation with a Linear Harmonic Chain
}

\author{
Yasusada Nambu* \\ Department of Physics, Graduate School of Science, \\ Nagoya University, Chikusa, Nagoya 464-8602, Japan \\ Masahiro Hottat \\ Department of Physics, Faculty of Science, \\ Tohoku University, Sendai 980-8578, Japan
}

(Dated: October 14, 2010)

\begin{abstract}
A protocol of quantum energy teleportation is proposed for a one-dimensional harmonic chain. A coherent-state positive operator-valued measure (POVM) measurement is performed on coupled oscillators of the chain in the ground state accompanied by energy infusion to the system. This measurement consumes a part of the ground state entanglement. Depending on the measurement result, a displacement operation is performed on a distant oscillator accompanied by energy extraction from the zero-point fluctuation of the oscillator. We find that the amount of consumed entanglement is bounded from below by a positive value that is proportional to the amount of teleported energy.
\end{abstract}

PACS numbers: 03.67.-a, 03.65.Ud

Keywords: entanglement; quantum energy teleportation, measurement, POVM

*Electronic address: nambu@gravity.phys.nagoya-u.ac.jp

${ }^{\dagger}$ Electronic address: hotta@tuhep.phys.tohoku.ac.jp 


\section{INTRODUCTION}

Recently, it has been reported that energy can be transported by local operations and classical communication (LOCC) while retaining local energy conservation and without breaking causality ( for spin systems [1 $[3]$, for trapped ion systems [4], and for quantum field systems [5-7]). Such protocols are called quantum energy teleportation (QET) and are based on ground-state entanglement of many-body quantum systems. By performing a local measurement on a subsystem $A$ of a many-body system in the ground state, information about the quantum fluctuation of $A$ can be extracted. During this measurement, some amount of energy is infused into $A$ as QET energy input, and the ground state entanglement gets partially broken. The measurement result is announced to another subsystem $B$ of the manybody system by the classical protocol. Using this information, energy can be extracted from $B$ by performing a local operation on $B$ dependent on the announced measurement data. The root of the protocols is a correlation between the measurement information of $A$ and the quantum fluctuation of $B$ via the ground state entanglement. The information about $A$ enables us to partially know what kind of zero-point fluctuation of $B$ is realized. Thus, using this information, we can select a good operation on $B$ for the energy extraction.

In general, we are able to make a better strategy for a task by obtaining more information. Hence, for the QET case, it sounds plausible to imagine that more information about the quantum fluctuation of $B$ is obtained by measurements of $A$, more energy can be teleported from $A$ to $B$. If we consume a large amount of ground-state entanglement between fluctuation of $A$ and fluctuation of $B$ during the measurement of $A$, it is naturally expected that much information about the post-measurement state of $B$ is included in $A$ 's measurement result. Therefore, there should exist some qualitative relationship between the breaking of entanglement by measurement and the amount of teleported energy of the optimal operation of $B$.

While the basic protocol of QET has been investigated for several model systems, the analysis of QET for many-body systems has not been development yet. This is because these quantities are difficult to obtain analytically for many-body systems. So far, the relation between teleported energy and entanglement breaking has been investigated only for the minimal model of QET [3] which consists of two qubits. In this paper, we aim to establish this relation of QET using a one-dimensional harmonic chain. There are many works in- 
vestigating the feature of entanglement in harmonic chain models [8 12]. The conventional method to analyze the entanglement in a harmonic chain is to introduce two spatial regions containing sites of the harmonic chain and consider the bipartite entanglement between these regions. Previous analysis shows that the zero temperature ground state (vacuum state) of the harmonic chain is entangled. Thus this entanglement can become a resource for QET. As we are not interested in the dynamical aspect of QET in the present analysis, we assumed that the speed of classical communication is infinity and our protocol can be treated as the non-relativistic one.

For a harmonic chain, we first consider the setting that groups $A$ and the $B$ consist of a single site. We investigate the relation between the amount of teleported energy and the quantum mutual information by changing the distance between $A$ and $B$. Then we consider the setting with a block of coupled harmonic oscillators as $A$ and its complementary set as $B$. Increasing the number of measured oscillators in $A$, we calculate both the entanglement breaking between $A$ and $B$ and the amount of energy teleported from $A$ to $B$ via a QET protocol. As a measure of entanglement, we use the logarithmic negativity [13]. An explicit inequality is given such that the breaking of entanglement is lower bounded by a positive value that is proportional to the amount of teleported energy. This shows how much entanglement is required to teleport energy in the harmonic chain.

The paper is organized as follows. In Sec. II, we introduce the harmonic chain model and we prepare a formula for the positive oeprator-valued measure (POVM) measurement for the harmonic chain in Sec. III. We present our numerical result in Sec. IV and Sec. V is devoted to a summary.

\section{HARMONIC CHAIN MODEL}

We consider a one-dimensional harmonic chain with $N$ sites. We assume that $N$ is an even number. The Hamiltonian is

$$
H=\frac{1}{2} \sum_{j=1}^{N}\left(p_{j}^{2}+q_{j}^{2}-\alpha q_{j} q_{j-1}\right), \quad(j=1, \cdots, N)
$$

where we assume a periodic boundary condition for $q_{j}, p_{j}$ and a positive parameter $\alpha \neq 1$ is introduced to regularize the infrared divergence which appears in the correlation matrix. $\alpha=1$ corresponds to the critical case (massless limit) of the harmonic chain. This criticality 
allows us to teleport energy to a distant point, decreasing the amount of energy obeying a power law decay with respect to the distance. As we will show, QET is possible for the non-critical case. However, the amount of teleported energy decreases exponentially with increasing distance and QET is not so effective. Thus, it is important to investigate QET with near-critical harmonic chains. The quantized canonical variables are

$$
\begin{aligned}
& q_{j}=\frac{1}{\sqrt{N}} \sum_{k=0}^{N-1}\left(f_{k} \hat{a}_{k}+f_{k}^{*} \hat{a}_{N-k}^{\dagger}\right) e^{i \theta_{k} j}, \\
& p_{j}=\frac{1}{\sqrt{N}} \sum_{k=0}^{N-1}(-i)\left(g_{k} \hat{a}_{k}-g_{k}^{*} \hat{a}_{N-k}^{\dagger}\right) e^{i \theta_{k} j}, \quad \theta_{k}=\frac{2 \pi k}{N}
\end{aligned}
$$

where $f_{k}, g_{k}$ are mode functions

$$
f_{k}=\frac{1}{\sqrt{2 \omega_{k}}} e^{-i \omega_{k} t}, \quad g_{k}=\sqrt{\frac{\omega_{k}}{2}} e^{-i \omega_{k} t}, \quad \omega_{k}^{2}=1-\alpha \cos \theta_{k}
$$

and the creation and annihilation operators satisfy

$$
\left[\hat{a}_{k}, \hat{a}_{k^{\prime}}^{\dagger}\right]=\delta_{k k^{\prime}}
$$

The two point correlation functions with respect the ground state satisfying $\hat{a}_{k}|g\rangle=0$ are

$$
\begin{aligned}
& \left\langle q_{i} q_{j}\right\rangle=\frac{1}{N} \sum_{k=0}^{N-1} \frac{1}{2 \omega_{k}} \cos \left[(i-j) \theta_{k}\right]=g_{|i-j|}=G_{i j} \\
& \left\langle p_{i} p_{j}\right\rangle=\frac{1}{N} \sum_{k=0}^{N-1} \frac{\omega_{k}}{2} \cos \left[(i-j) \theta_{k}\right]=h_{|i-j|}=H_{i j} \\
& \left\langle q_{i} p_{j}\right\rangle=\frac{i}{2 N} \sum_{k=0}^{N-1} e^{i \theta_{k}(i-j)}=\frac{i}{2} \delta_{i j}, \\
& \left\langle q_{i}\right\rangle=\left\langle p_{i}\right\rangle=0
\end{aligned}
$$

where we have introduced the matrices $G$ and $H$ satisfying the relation

$$
(G H)_{i j}=\frac{1}{4} \delta_{i j}
$$

As the ground state is a Gaussian state, it can be completely determined using these two point correlation functions.

\section{POVM MEASUREMENT AND ENERGY TELEPORTATION}

We briefly review the protocol of QET (Fig. 1). In these figures, quantum fluctuations in the harmonic chain are schematically shown as broken lines. The dashed horizontal lines 
represent the magnitude of quantum fluctuation of the state. The protocol consists of the following steps: 
( 1$)$

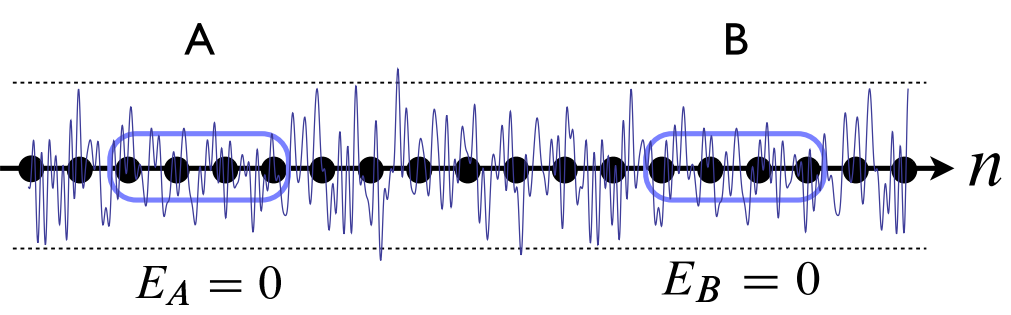

(2) measurement of $A$
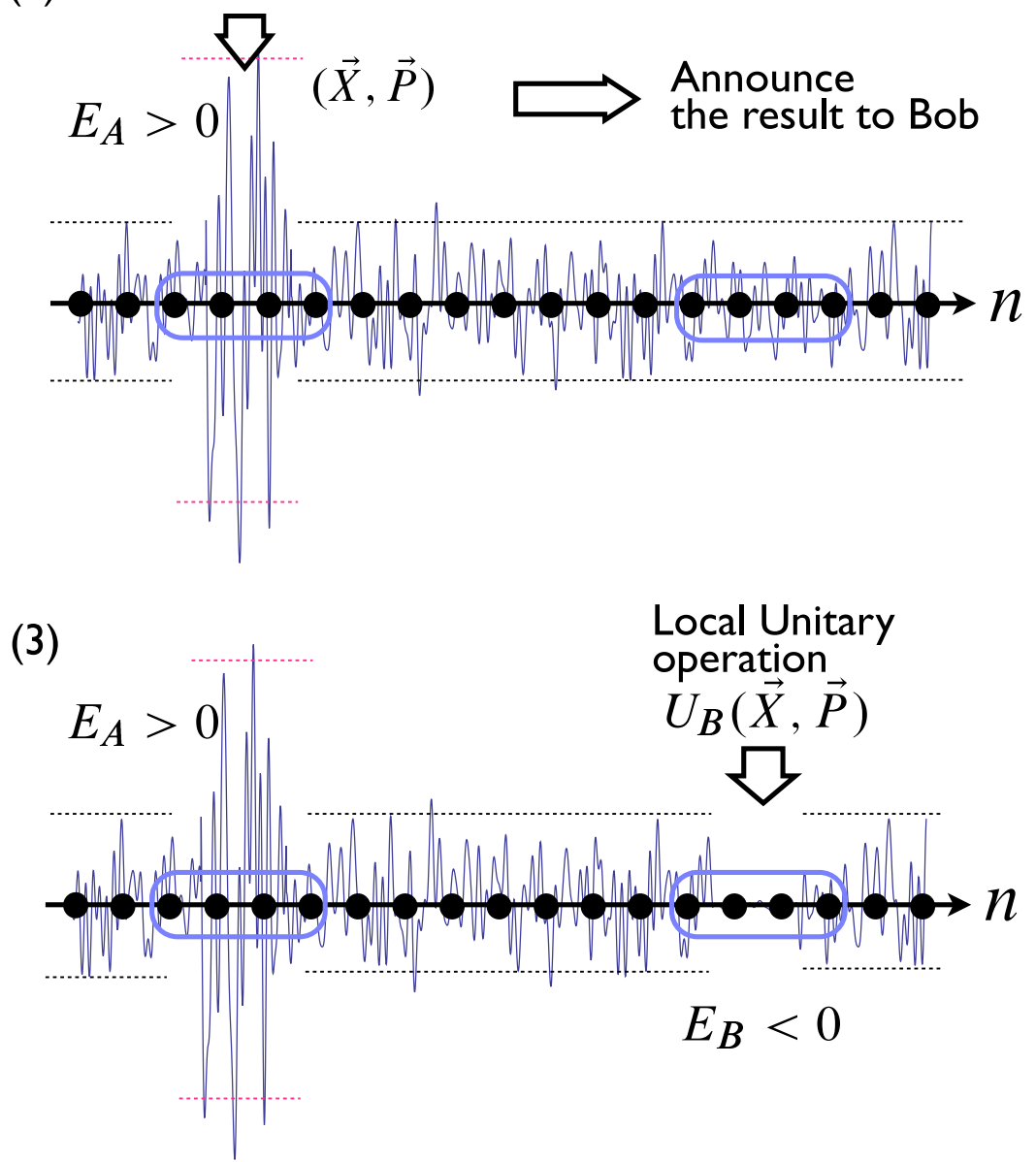

FIG. 1: The protocol of QET with a harmonic chain. (1) We prepare the ground state of a harmonic chain and introduce two parties $A$ and $B$. The local energy of $A$ and $B$ is zero. (2) The measurement of $A$ increases the energy of the group $A$. The result of the measurement is announced to Bob via classical communication. (3) Depending on the announced measurement result, Bob performs a local unitary operation on the group $B$, which reduces the energy of the group $B$ so that it becomes negative. The dashed horizontal lines represent the magnitude of quantum fluctuation of the state. 
(1) We prepare the ground state of the harmonic chain and define groups $A$ and $B$ of coupled oscillators in the system. $A$ and $B$ consist of some number of sites. The groups $A$ and $B$ are entangled due to the entanglement of the ground state quantum fluctuation of the harmonic chain. This entanglement enables us to extract a positive energy via a protocol of QET.

(2) Alice makes a local measurement of quantum fluctuations of sites in the group $A$. Because the post-measurement state is not the ground state, it is an excited state with positive energy. As some amount of energy is infused through this measurement procedure, the energy of the group $A$ after the measurement becomes positive provided that the ground state energy before the measurement is chosen to be zero. By this measurement, Alice

obtains measurement results for sites in the group $A$. She announces these values to Bob via classical communication.

(3) Depending on the announced measurement results, Bob performs a local unitary operation on sites in the group $B$. By choosing the operation suitably to suppress the zeropoint fluctuation of $B$, it can be shown that the local energy of the group $B$ takes a negative value lower than the ground-state one. Thus we can extract positive energy from the system.

In our numerical investigation of QET for the harmonic chain, we apply a POVM measurement [14] to sites in the group $A$ (see Fig. 2 and 6). The measurement reduces the entanglement between the two groups. What we want to know is the relation between the amount of entanglement breaking due to the measurement and the extracted energy via the protocol of QET.

\section{A. The state before and after the measurement}

We assume that the state of the system before the measurement is the ground state. In the coordinate representation, the state is given by

$$
\rho_{0}=\langle\vec{q} \mid g\rangle\left\langle g \mid \vec{q}^{\prime}\right\rangle, \quad \vec{q}=\left(q_{1}, \cdots, q_{N}\right)^{T}
$$

where

$$
\langle\vec{q} \mid g\rangle \propto \exp \left[-\frac{1}{4} \sum_{i, j=1}^{N} q_{i}\left(G^{-1}\right)_{i j} q_{j}\right]=\exp \left[-\sum_{i, j=1}^{N} q_{i} H_{i j} q_{j}\right] .
$$

We apply a coherent-state POVM measurement to $2 \ell+1$ sites in the group $A$. To define the POVM adopted in our analysis, let us introduce the following annihilation operator for 
the canonical variable $\left(q_{n}, p_{n}\right)$ of the site $n$ in the group $A$ :

$$
\hat{b}_{n}=\sqrt{\frac{\omega}{2}} \hat{q}_{n}+\frac{i}{\sqrt{2 \omega}} \hat{p}_{n}, \quad 1 \leq n \leq 2 \ell+1 .
$$

We define the ground state $\left|0_{n}\right\rangle$ with respect to the operator $\hat{b}_{n}$ as

$$
\hat{b}_{n}\left|0_{n}\right\rangle=0
$$

and the coherent state defined by $\hat{b}_{n}^{\dagger}$ as

$$
\left|X_{n}, P_{n}\right\rangle=e^{-\left|c_{n}\right|^{2} / 2} \exp \left(c_{n} \hat{b}_{n}^{\dagger}\right)\left|0_{n}\right\rangle, \quad c_{n}=\sqrt{\frac{\omega}{2}} X_{n}+\frac{i}{\sqrt{2 \omega}} P_{n},
$$

where $X_{n}$ and $P_{n}$ are real numbers and are classical amplitude of the coherent state. The expectation values of site variables with respect to this coherent state are

$$
\begin{aligned}
& \left\langle X_{n}, P_{n}\left|q_{n}\right| X_{n}, P_{n}\right\rangle=X_{n}, \quad\left\langle X_{n}, P_{n}\left|p_{n}\right| X_{n}, P_{n}\right\rangle=P_{n} \\
& \left\langle X_{n}, P_{n}\left|q_{n}^{2}\right| X_{n}, P_{n}\right\rangle=X_{n}^{2}+\frac{1}{2 \omega}, \quad\left\langle X_{n}, P_{n}\left|p_{n}^{2}\right| X_{n}, P_{n}\right\rangle=P_{n}^{2}+\frac{\omega}{2} .
\end{aligned}
$$

In the coordinate representation, the coherent state can be written

$$
\left\langle q_{n} \mid X_{n}, P_{n}\right\rangle=\left(\frac{\omega}{\pi}\right)^{1 / 4} \exp \left[-\frac{\omega}{2}\left(q_{n}-X_{n}\right)^{2}+i P_{n}\left(q_{n}-X_{n}\right)\right] .
$$

The measurement operator for the POVM is given by

$$
M_{\ell}(\vec{X}, \vec{P})=\frac{1}{(\sqrt{2 \pi})^{2 \ell+1}} \prod_{n=1}^{2 \ell+1}\left|X_{n}, P_{n}\right\rangle\left\langle X_{n}, P_{n}\right|, \quad 1 \leq \ell \leq \frac{N}{2}-2
$$

where

$$
\vec{X}=\left(X_{1}, \cdots, X_{2 \ell+1}\right)^{T}, \quad \vec{P}=\left(P_{1}, \cdots, P_{2 \ell+1}\right)^{T} .
$$

The POVM is defined by

$$
\Pi_{\ell}(\vec{X}, \vec{P})=M_{\ell}^{\dagger}(\vec{X}, \vec{P}) M_{\ell}(\vec{X}, \vec{P})=\frac{1}{(2 \pi)^{2 \ell+1}} \prod_{n=1}^{2 \ell+1}\left|X_{n}, P_{n}\right\rangle\left\langle X_{n}, P_{n}\right|
$$

and satisfies the following completeness relation for the measurement operator:

$$
\int\left(\prod_{n=1}^{2 \ell+1} d X_{n} d P_{n}\right) \Pi_{\ell}(\vec{X}, \vec{P})=I
$$

If we obtain the value $(\vec{X}, \vec{P})$ as the result of the POVM measurement, the state after the measurement can be written as

$$
\rho_{\ell}(\vec{X}, \vec{P})=\prod_{n=1}^{2 \ell+1}\left\langle X_{n}, P_{n} \mid g\right\rangle\left\langle g \mid X_{n}, P_{n}\right\rangle \otimes \prod_{n^{\prime}=1}^{2 \ell+1}\left|X_{n^{\prime}}, P_{n^{\prime}}\right\rangle\left\langle X_{n^{\prime}}, P_{n^{\prime}}\right| .
$$


After the measurement, the states for the measured sites become the coherent states (9) and states for other sites are computed by acting the measurement projection operator on the ground state. To obtain the explicit form of the state after the POVM measurement, we introduce the following vector notation for the site variables:

$$
\left(q_{n}\right)=\left(\begin{array}{c}
\vec{q}_{\ell}^{M} \\
\vec{q}_{\ell}
\end{array}\right), \quad\left(p_{n}\right)=\left(\begin{array}{c}
\vec{p}_{\ell}^{M} \\
\vec{p}_{\ell}
\end{array}\right) .
$$

The $(2 \ell+1)$-dimensional vectors $\vec{q}_{\ell}^{M}, \vec{p}_{\ell}^{M}$ represent variables for the sites whose values are measured. Then, with respect to these bases, the correlation matrices (44) and (5) for site variables can be written

$$
H=\left(\begin{array}{cc}
L_{\ell} & K_{\ell} \\
K_{\ell}^{T} & H_{\ell}
\end{array}\right), \quad G=\left(\begin{array}{cc}
C_{\ell} & D_{\ell} \\
D_{\ell}^{T} & G_{\ell}
\end{array}\right),
$$

where $L_{\ell}, C_{\ell}$ denote the $(2 \ell+1) \times(2 \ell+1)$ correlation matrices for the measured sites. Using this notation, the ground state projected to the coherent state (9) is given by

$$
\begin{aligned}
\langle\vec{X}, \vec{P} \mid g\rangle \propto \exp [- & \vec{q}_{\ell}^{T}\left(H_{\ell}-K_{\ell}^{T}\left(L_{\ell}+\frac{\omega}{2}\right)^{-1} K_{\ell}\right) \vec{q}_{\ell}+\vec{q}_{\ell}^{T} K_{\ell}^{T}\left(L_{\ell}+\frac{\omega}{2}\right)^{-1}(i \vec{P}-\omega \vec{X}) \\
& -\frac{\omega}{2} \vec{X}^{T}\left(1-\frac{\omega}{2}\left(L_{\ell}+\frac{\omega}{2}\right)^{-1}\right) \vec{X}-\frac{1}{4} \vec{P}^{T}\left(L_{\ell}+\frac{\omega}{2}\right)^{-1} \vec{P} \\
& \left.+\frac{i}{2} \vec{P}^{T}\left(1-\frac{\omega}{2}\left(L_{\ell}+\frac{\omega}{2}\right)^{-1}\right) \vec{X}+\frac{i}{2} \vec{X}^{T}\left(1-\frac{\omega}{2}\left(L_{\ell}+\frac{\omega}{2}\right)^{-1}\right) \vec{P}\right] .
\end{aligned}
$$

The two-point correlation functions for fluctuations $\Delta q_{i}=q_{i}-\left\langle q_{i}\right\rangle, \Delta p_{i}=p_{i}-\left\langle p_{i}\right\rangle$ after the measurement are obtained by taking the expectation value with respect to the state (14). The non-zero correlation functions are

$$
\begin{aligned}
i, j \in(\text { measured site in } A): & \left\langle\Delta q_{i} \Delta q_{j}\right\rangle=\frac{\delta_{i j}}{2 \omega}, \quad\left\langle\Delta p_{i} \Delta p_{j}\right\rangle=\frac{\omega}{2} \delta_{i j}, \\
& \left\langle\Delta p_{i} \Delta q_{i}+\Delta q_{i} \Delta p_{i}\right\rangle=0 \\
i, j \notin(\text { measured site in } A): & \left\langle\Delta q_{i} \Delta q_{j}\right\rangle=\frac{1}{4}\left(M^{-1}\right)_{i-(2 \ell+1), j-(2 \ell+1)}, \\
& \left\langle\Delta p_{i} \Delta p_{j}\right\rangle=(M)_{i-(2 \ell+1), j-(2 \ell+1)}
\end{aligned}
$$

where the matrix $M$ is defined by

$$
M=H_{\ell}-K_{\ell}^{T}\left(L_{\ell}+\frac{\omega}{2}\right)^{-1} K_{\ell} .
$$


The other two-point functions are zero. These covariances are independent of the result of the measurement $(\vec{X}, \vec{P})$. The probability distribution to obtain the measured value $(\vec{X}, \vec{P})$ is given by

$$
\begin{aligned}
\left\langle g\left|\Pi_{\ell}(\vec{X}, \vec{P})\right| g\right\rangle & =\int d \vec{q}_{\ell}|\langle g \mid \vec{X}, \vec{P}\rangle|^{2} \\
& \propto \exp \left[-\frac{1}{2} \vec{P}^{T}\left(L_{\ell}+\frac{\omega}{2}\right)^{-1} \vec{P}-\frac{1}{2} \vec{X}^{T}\left(C_{\ell}+\frac{1}{2 \omega}\right)^{-1} \vec{X}\right]
\end{aligned}
$$

\section{B. The optimized energy}

We define the energy of the lattice site $n$ as

$$
H_{n}=\frac{1}{2}\left[p_{n}^{2}+q_{n}^{2}-\frac{\alpha}{2} q_{n}\left(q_{n-1}+q_{n+1}\right)-\epsilon\right]
$$

where the constant $\epsilon$ is chosen to satisfy

$$
\left\langle g\left|H_{n}\right| g\right\rangle=0
$$

After the measurement, we obtain the values $(\vec{X}, \vec{P})$ of the measured sites. Depending on these measured values, we apply the following local displacement operation to sites in the group $B$ which is composed of a single site in our numerical setting $\left(n_{B}=N / 2+\ell+1\right)$

$$
U_{B}(\vec{X}, \vec{P})=\exp \left[i\left((\vec{\theta} \cdot \vec{P}) q_{n_{B}}-(\vec{\phi} \cdot \vec{X}) p_{n_{B}}\right)\right]
$$

where $\vec{\theta}$ and $\vec{\phi}$ are constant parameters to be determined later by optimization. Then, the transformed state is

$$
\rho_{\ell}^{\prime}=\int \prod_{n=1}^{2 \ell+1}\left(d X_{n} d P_{n}\right) U_{B}(\vec{X}, \vec{P}) M_{d}(\vec{X}, \vec{P})|g\rangle\langle g| M_{d}^{\dagger}(\vec{X}, \vec{P}) U_{B}^{\dagger}(\vec{X}, \vec{P}) .
$$

The energy of the site $B$ with respect to this state is

$$
\begin{aligned}
\left\langle H_{B}\right\rangle= & \sum_{j=1}^{2 \ell+1} \theta_{j}\left\langle g\left|p_{j} p_{n_{B}}\right| g\right\rangle+\sum_{j=1}^{2 \ell+1} \phi_{j}\left\langle g\left|q_{j}\left(q_{n_{B}}-\frac{\alpha}{2}\left(q_{n_{B}+1}+q_{n_{B}-1}\right)\right)\right| g\right\rangle \\
& \quad+\frac{1}{2} \sum_{j, k=1}^{2 \ell+1} \theta_{j}\left(\left\langle g\left|p_{j} p_{k}\right| g\right\rangle+\frac{\omega}{2} \delta_{j k}\right) \theta_{k}+\frac{1}{2} \sum_{j, k=1}^{2 \ell+1} \phi_{j}\left(\left\langle g\left|q_{j} q_{k}\right| g\right\rangle+\frac{1}{2 \omega}\right) \phi_{k} \\
= & \frac{1}{2} \vec{\theta}^{T} T_{p} \vec{\theta}+\overrightarrow{J_{p}} \cdot \vec{\theta}+\frac{1}{2} \vec{\phi}^{T} T_{q} \vec{\phi}+\overrightarrow{J_{q}} \cdot \vec{\phi}
\end{aligned}
$$


where we have introduced

$$
\begin{aligned}
& \left(T_{p}\right)_{j k}=h_{|j-k|}+\frac{\omega}{2} \delta_{j k}, \quad\left(T_{q}\right)_{j k}=g_{|j-k|}+\frac{1}{2 \omega} \delta_{j k}, \\
& \left(\overrightarrow{J_{p}}\right)_{j}=h_{\left|j-n_{B}\right|}, \quad\left(\overrightarrow{J_{q}}\right)_{j}=g_{\left|j-n_{B}\right|}-\frac{\alpha}{2}\left(g_{\left|j-\left(n_{B}-1\right)\right|}+g_{\left|j-\left(n_{B}+1\right)\right|}\right) .
\end{aligned}
$$

By choosing the following values of the parameters

$$
\vec{\theta}=-T_{p}^{-1} \vec{J}_{p}, \quad \vec{\phi}=-T_{q}^{-1} \vec{J}_{q}
$$

we obtain the following minimum (optimized) value of the site $B$ 's energy

$$
\left\langle H_{B}\right\rangle_{\mathrm{opt}}=-\frac{1}{2} \vec{J}_{p}^{T} T_{p}^{-1} \vec{J}_{p}-\frac{1}{2} \vec{J}_{q}^{T} T_{q}^{-1} \vec{J}_{q}<0
$$

The negative sign of $\left\langle H_{B}\right\rangle$ means that a positive amount of energy is transferred from the chain to outside, obeying local energy conservation.

\section{Bipartite entanglement and mutual information}

To investigate the relation between the amount of extractable energy via QET and the entanglement breaking due to the measurement, we consider the bipartite entanglement between the group $A$ and the group $B$. For this purpose, we use the logarithmic negativity $E_{N}$ as our measure of entanglement. The logarithmic negativity provides an upper bound on the efficiency of distillation of entanglement [13].

The canonical variables of our bipartite system are

$$
\vec{\xi}^{T}=\left(\vec{q}_{A}, \vec{p}_{A}, q_{B}, p_{B}\right), \quad\left[\xi_{j}, \xi_{k}\right]=i \Omega_{j k}, \quad j, k=1, \cdots, 2 N
$$

where $\Omega$ is a $2 N \times 2 N$ matrix

$$
\boldsymbol{\Omega}=\left(\begin{array}{ccc}
\boldsymbol{J} & & \\
& \ddots & \\
& & \boldsymbol{J}
\end{array}\right), \quad \boldsymbol{J}=\left(\begin{array}{cc}
0 & 1 \\
-1 & 0
\end{array}\right) .
$$

As we are assuming a Gaussian state, the state is completely specified by the following covariance matrix

$$
V_{j k}=\frac{1}{2}\left\langle\xi_{j} \xi_{k}+\xi_{k} \xi_{j}\right\rangle-\left\langle\xi_{j}\right\rangle\left\langle\xi_{k}\right\rangle
$$


The symplectic eigenvalue $\nu_{n}$ of the covariance matrix $\boldsymbol{V}$ satisfies the inequality

$$
\nu_{n} \geq \frac{1}{2}
$$

which is the generalization of the uncertainty relation. The logarithmic negativity is defined by the partially transposed covariance matrix $\tilde{\boldsymbol{V}}$ obtained by reversing the sign of party $B$ 's momentum

$$
E_{N} \equiv-\sum_{n=1}^{N} \min \left[0, \log _{2}\left(2 \tilde{\nu}_{n}\right)\right]
$$

where $\tilde{\nu}_{n}$ is the symplectic eigenvalue of the partially transposed covariance matrix $\tilde{\boldsymbol{V}}$. If this quantity is positive, the bipartite system is entangled, and we can use the logarithmic negativity as the measure of the bipartite entanglement between groups $A$ and $B$. The logarithmic negativity provides the sufficient condition for entanglement between $A$ and $B$.

We also consider the quantum mutual information of $A$ and $B$ as a measure of correlation between $A$ and $B$. For the Gaussian system with the covariance matrix $\boldsymbol{V}$, in terms of symplectic eigenvalues $\nu_{n}$, the von Neuman entropy of the system is given by [15]

$$
S=\sum_{n} f\left(\nu_{n}\right), \quad f(x)=\left(x+\frac{1}{2}\right) \ln \left(x+\frac{1}{2}\right)-\left(x-\frac{1}{2}\right) \ln \left(x-\frac{1}{2}\right) .
$$

For the bipartite system with the group $A$ and $B$, the mutual information is defined by

$$
S_{M}=S(A)+S(B)-S(A+B)
$$

This quantity represents the total correlations including the quantum and the classical correlation between the group $A$ and $B$ [16]. A large value of $S_{M}$ implies that $A$ and $B$ are strongly correlated and we are able to obtain much information about $B$ just by measuring $A$. Thus, this is expected to assure a large amount of teleported energy.

We numerically calculate the logarithmic negativity and the mutual information. The covariance matrix of the total system before the measurement is

$$
V_{0}=\left(\begin{array}{ccc}
U_{0}^{(1,1)} & \ldots & U_{0}^{(1, N)} \\
\vdots & \ddots & \vdots \\
U_{0}^{(N, 1)} & \ldots & U_{0}^{(N, N)}
\end{array}\right), \quad U_{0}^{(i, j)}=\left(\begin{array}{cc}
\left\langle\Delta q_{i} \Delta q_{j}\right\rangle & 0 \\
0 & \left\langle\Delta p_{i} \Delta p_{j}\right\rangle
\end{array}\right)=\left(\begin{array}{cc}
g_{|i-j|} & 0 \\
0 & h_{|i-j|}
\end{array}\right)
$$

The covariance matrix after the measurement is

$$
V^{M}=\left(\begin{array}{cc}
V_{A}^{M} & 0 \\
0 & V_{A B}
\end{array}\right)
$$


where $V_{A}^{M}$ represents the covariance matrix for the measured sites and is given by the $2(2 \ell+1) \times 2(2 \ell+1)$ diagonal matrix

$$
V_{A}^{M}=\left(\begin{array}{ccc}
U_{1} & & \\
& \ddots & \\
& & U_{1}
\end{array}\right), \quad U_{1}=\left(\begin{array}{cc}
\frac{1}{2 \omega} & 0 \\
0 & \frac{\omega}{2}
\end{array}\right)
$$

and $V_{A B}$ represents the covariance matrix for the un-measured sites,

$$
V_{A B}=\left(\begin{array}{ccc}
U^{(1,1)} & \ldots & U^{(1, N-2 d-1)} \\
\vdots & \ddots & \vdots \\
U^{(N-2 d-1,1)} & \ldots & U^{(N-2 d-1, N-2 d-1)}
\end{array}\right), \quad U^{(i, j)}=\left(\begin{array}{cc}
\frac{1}{4}\left(M^{-1}\right)_{i, j} & 0 \\
0 & (M)_{i, j}
\end{array}\right)
$$

By use of these expressions for the covariance matrices, the logarithmic negativity and the mutual information before and after the measurement can be obtained numerically.

\section{NUMERICAL RESULT}

We numerically calculated the decrease of the logarithmic negativity due to the measurement

$$
\Delta E_{N}=E_{N}(\text { before measurement })-E_{N}(\text { after measurement })
$$

and the optimized energy of the site $B$ obtained via the protocol of QET. We consider the two different settings of numerical calculations to establish the relation between the entanglement breaking and the amount of teleported energy. As the parameter $\alpha$ of the harmonic chain, we used the following four values:

$$
\alpha_{1}=0.90, \quad \alpha_{2}=0.95, \quad \alpha_{3}=0.99, \quad \alpha_{4}=1-10^{-7} \text { (critical). }
$$

We have also investigated harmonic chains with smaller values of $\alpha$, but the amount of teleported energy is reduced and QET is not effective for small values of $\alpha$. We adopt these four values of $\alpha$ as typical cases of our numerical calculation. The values $\alpha_{1}, \alpha_{2}, \alpha_{3}$ represent typical examples of the non-critical case and $\alpha_{4}$ represents the critical case with a small cutoff parameter $10^{-7}$ which is necessary to evaluate correlation matrices numerically. As we will see later, the numerically obtained quantities converge to the value with $\alpha_{4}$ as the value of $\alpha$ approaches 1 . 


\section{A. Setting 1}

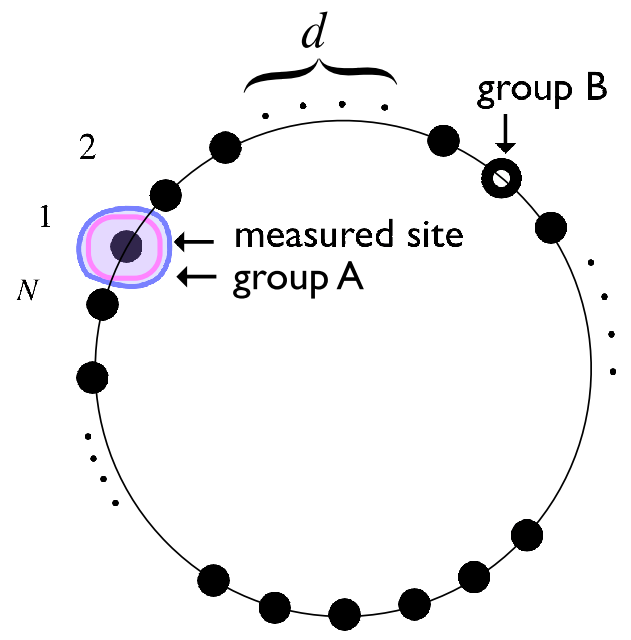

FIG. 2: The setting 1 of our numerical calculation. We change the separation $d$ between sites $A$ and $B$.

As the first setting of our numerical calculation, group $A$ and group $B$ consist of a single site (Fig. 2). We fix the total site number $N=100$. We apply the POVM measurement to the site $A$. By changing the separation $d$ between $A$ and $B$, we observe how the entanglement between the two groups and the optimized energy (26) of the site $B$ change.
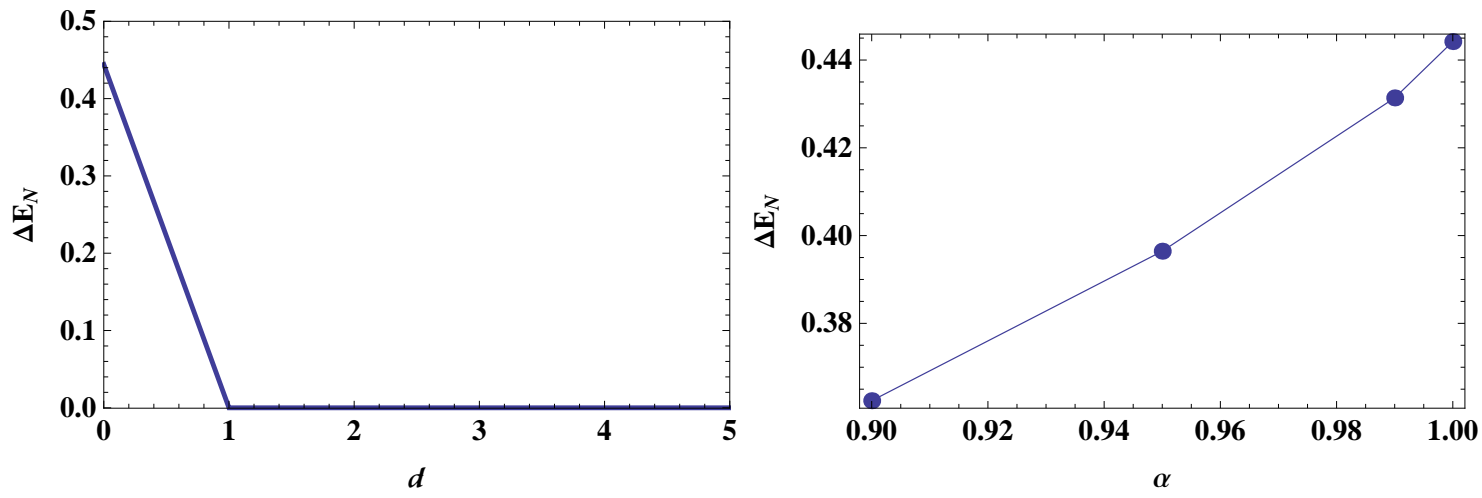

FIG. 3: Left panel: $d$ dependence of the decrease of the logarithmic negativity due to the measurement for $\alpha_{4}=1-10^{-7}$. The logarithmic negativity before and after the measurement is zero for $d \geq 1$ and this behavior is the same for the other values of $\alpha$. Right panel: $\alpha$ dependence of the $\Delta E_{N}$ for $d=0$.

Figure 3 shows $\Delta E_{N}$ as a function of the separation between $A$ and $B$. The bipartite 
system composed of $A$ and $B$ is entangled only for $d=0$ before the measurement. After the measurement, $A$ and $B$ become separable even for $d=0$. This behavior is the same for other values of $\alpha$. As $\alpha$ approaches 1, the entanglement consumed via measurement increases. Our numerical calculation indicates that we have no entanglement breaking for $d=1,2,3, \cdots$. However, $\Delta E_{N}=0$ for $d \neq 0$ does not mean sites $A$ and $B$ do not have the correlation necessary to establish the QET protocol.

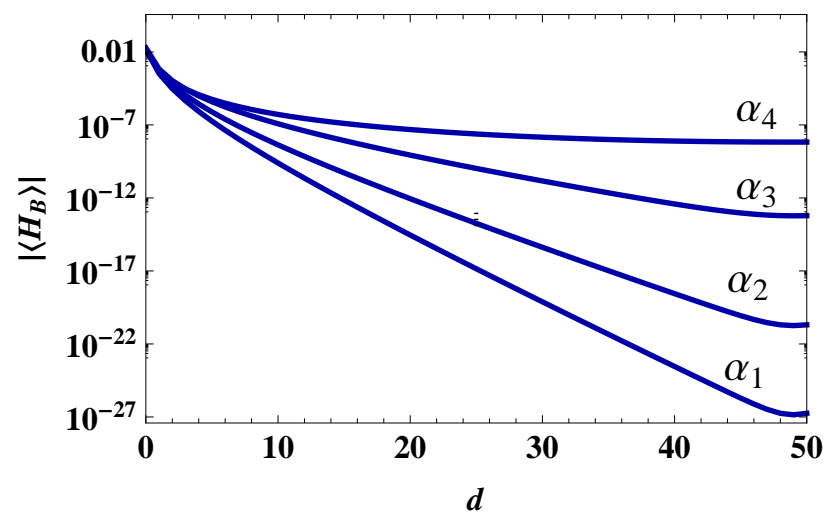

FIG. 4: $d$ dependence of the optimized energy of the site $B$. For the critical case $\alpha=\alpha_{4}$, this quantity behaves as $\left|\left\langle H_{B}\right\rangle\right| \sim 2 \times 10^{-3} d^{-3.6}$ for large $d$.

Indeed, as Fig. 4 shows, we have non-zero (negative) optimized energy of the site $B$ even for $\Delta E_{N}=0(d \geq 1)$. The amount of optimized energy of site $B$ decreases as $d$ increases. For the critical case $\alpha_{4}$, the optimized energy for large $d$ behaves as

$$
\left|\left\langle H_{B}\right\rangle\right| \sim 2 \times 10^{-3} d^{-3.6}
$$

To understand the behavior of the optimized energy from the view point of entanglement breaking, we investigated the $d$ dependence of the mutual information (31) (Fig. [5). 




FIG. 5: $d$ dependence of the decrease of the mutual information due to the measurement. For the critical case $\alpha=\alpha_{4}$, this quantity behaves as $\Delta S_{M} \sim 1.55 d^{-0.11}$ for large $d$.

The mutual information represents the total correlation of the bipartite system including the quantum and the classical correlations [16]. This quantity is suitable for understanding the behavior of QET for the setting 1 because the decrease of the logarithmic negativity becomes zero for $d \geq 1$, and it seems that we do not have any quantum correlations between $A$ and $B$. In this case, the information on the entanglement of the ground state is encoded as classical correlation between $A$ and $B$, which is established after the POVM measurement of $A$. As $d$ increases, the mutual information monotonically decreases. For the critical case, we have

$$
\Delta S_{M} \sim 1.55 d^{-0.11}
$$

The behavior of the mutual information is consistent with the $d$ dependence of the optimized energy; the larger the amount of the mutual information, the larger the amount of the optimized energy. Thus, we can conclude that the amount of energy teleported via the protocol of QET is related to the amount of breaking of the correlation between the group $A$ and $B$. Here it should be stressed that the "classical" correlation supporting energy teleportation is induced by ground-state entanglement which is purely quantum. If we have no entanglement in the ground state, we do not have any correlation between $A$ and $B$.

For the critical case, we obtained the scaling behavior (37) and (38) numerically. However, we have not developed any theoretical explanation for the values of these power law indices. We expect that an analysis based on conformal field theory may reveal these values. 


\section{B. Setting 2}

In this setting, the group $A$ consists of $N-1$ sites and the group $B$ is its complement and consists of a single site (Fig. 6).

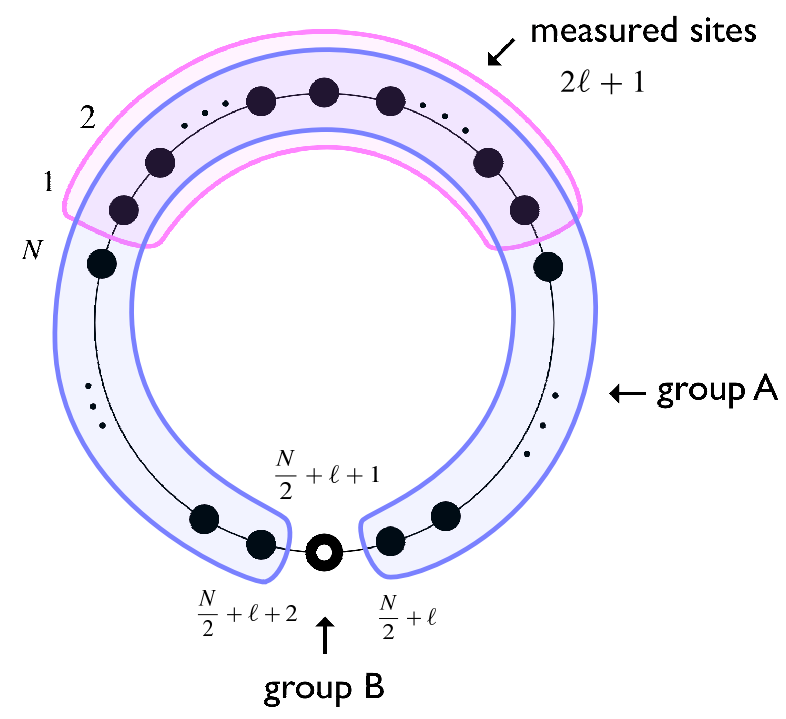

FIG. 6: The setting 2 of our numerical calculation. We change the number of measured sites in group $A$.

We change the number $2 \ell+1$ of measured sites in group $A$. As we explained in Sec. III, the procedure of the measurement infuses energy into the system. Hence, as $\ell$ increases, the state of the bipartite system after the measurement is strongly affected by the measurement process. In this sense, we can control the strength of the measurement from "weak" to "strong" by increasing the number of measured sites $\ell$ in the group $A$. We expect that the stronger the measurement becomes, the larger the amount of entanglement breaking and this enables us to obtain a larger amount of teleported energy of the site $B$ via the protocol of QET. 

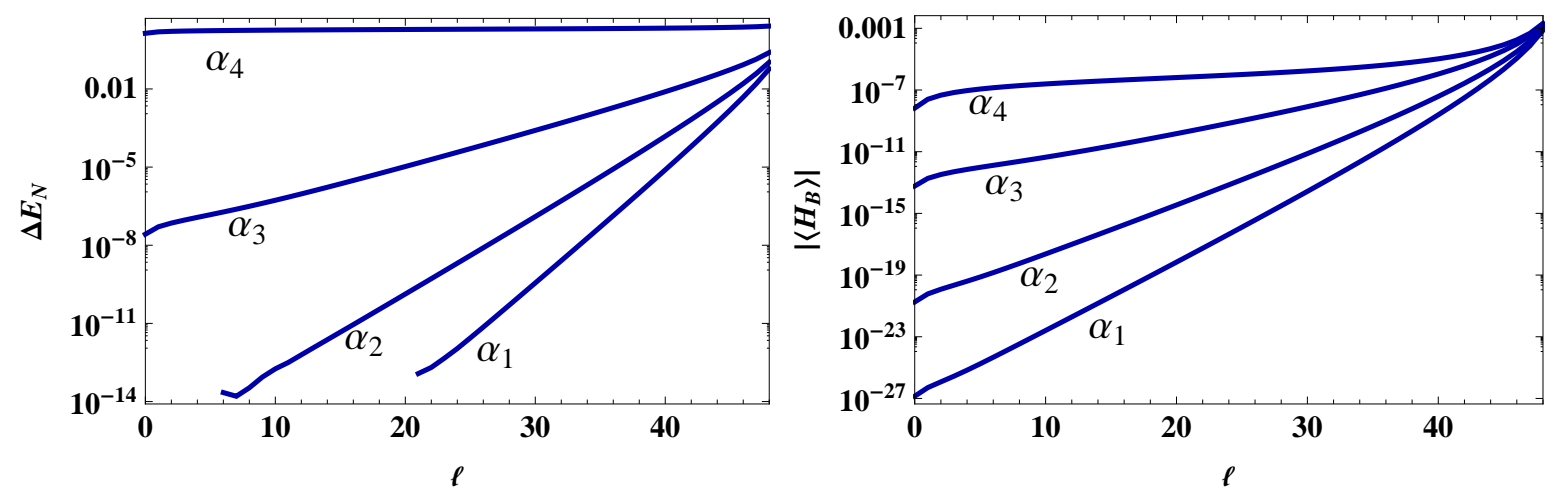

FIG. 7: Left panel: decrease of the logarithmic negativity due to the measurement. Right panel: optimized energy of site $B$.

Figure 7 shows the decrease of the logarithmic negativity due to the measurement, and the optimized energy obtained for the site $B$ via QET for the system size $N=100$. We have also investigated the system sizes $2 \leq N \leq 100$ and confirmed that the behavior of these quantities is same. Hence, we present the result with $N=100$. As the measurement becomes "strong" ( $\ell$ increases), the amount of entanglement breaking and the resulting optimized energy of site $B$ increases. In this setting, as the total bipartite system is pure state, the system becomes separable after the measurement when the all sites in $A$ are measured and the entanglement breaking due to the measurement is maximal. This behavior is consistent with our naive expectation that the ground- state entanglement of the harmonic chain is a resource for QET. Figure 8 is the $\ell$ dependence of the ratio of the optimized energy of site $B$ and the entanglement breaking. 


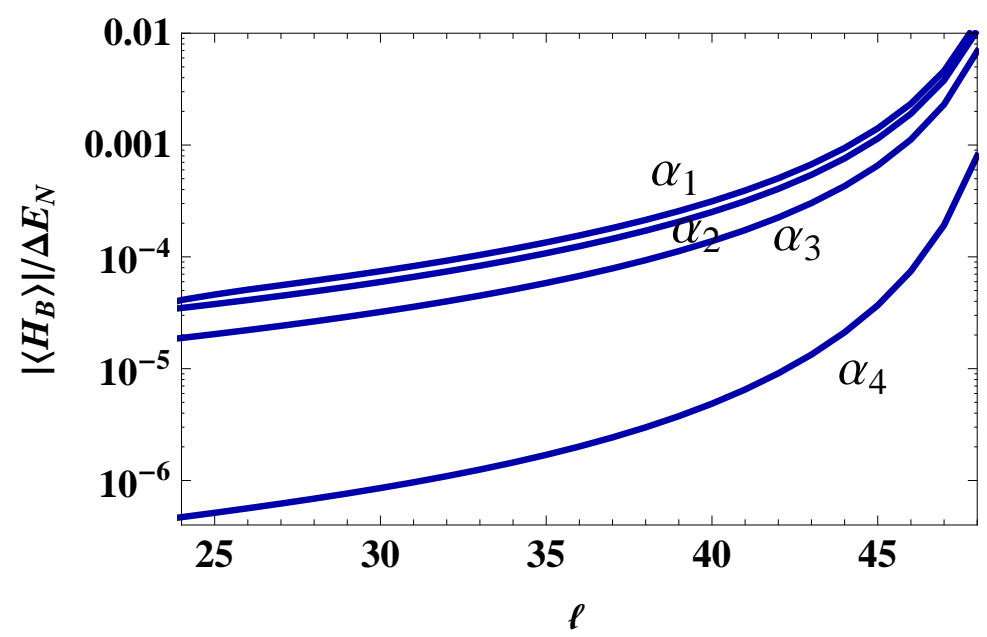

FIG. 8: Ratio of the optimized energy of site $B$ and the amount of entanglement breaking.

As we can observe, this ratio is monotonically increasing with respect to the size of the measured site $\ell$ and bounded from above. The maximum value of the ratio is achieved at $\ell=N / 2-2$, which is the maximal number of the measuring sites in group $A$. Thus, the maximal amount of teleported energy is bounded by the amount of entanglement breaking and the following inequality holds:

$$
\left|\left\langle H_{B}\right\rangle\right|<\beta \Delta E_{N}, \quad \beta<1,
$$

where $\beta$ is a constant whose value depends on the system size $N$ and the parameter $\alpha$. To find the relation between the amount of entanglement breaking and the optimized energy, we investigated the $N$ dependence of $\Delta E_{N}$ and $\left\langle H_{B}\right\rangle$ for a specific value of $\ell=N / 2-2$; this value of $\ell$ corresponds to the maximal possible number of measuring sites in group $A$ for a given $N$. The entanglement breaking due to the measurement is expected to become maximal (the strongest measurement case).

As the system size $N$ increases, the amount of entanglement breaking and the teleported energy both decrease (Fig. 9). 

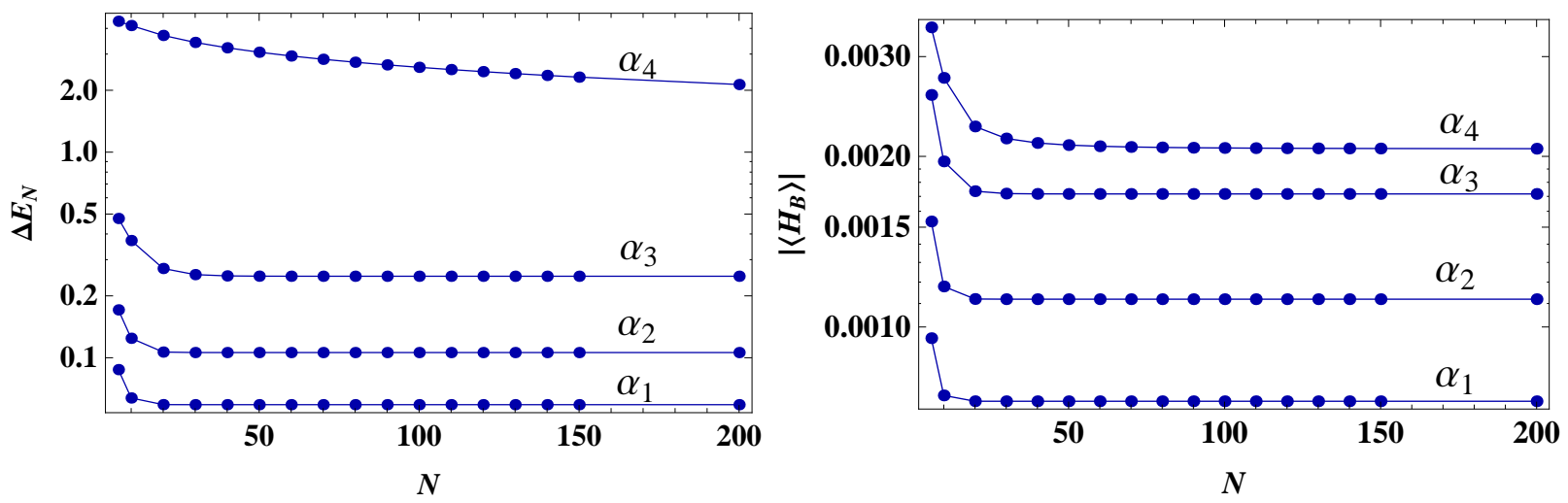

FIG. 9: $N$ dependence of $\Delta E_{N}$ and $\left|\left\langle H_{B}\right\rangle\right|$ for $\ell=N / 2-2$.

For the non-critical case $\alpha=\alpha_{1}, \alpha_{2}, \alpha_{3}$, these quantities approach constant values for large $N$. For the critical case $\alpha=\alpha_{4}$, we have the following scaling behavior

$$
\Delta E_{N} \sim 8 N^{-0.32}, \quad\left|\left\langle H_{B}\right\rangle\right| \sim 0.12 N^{-2.1}+0.0020613
$$

By taking the ratio of these quantities, we found that the following inequality holds:

$$
\left|\left\langle H_{B}\right\rangle\right|<\beta(N) \Delta E_{N}
$$

The behavior of the function $\beta(N)$ is shown in Fig. 10.

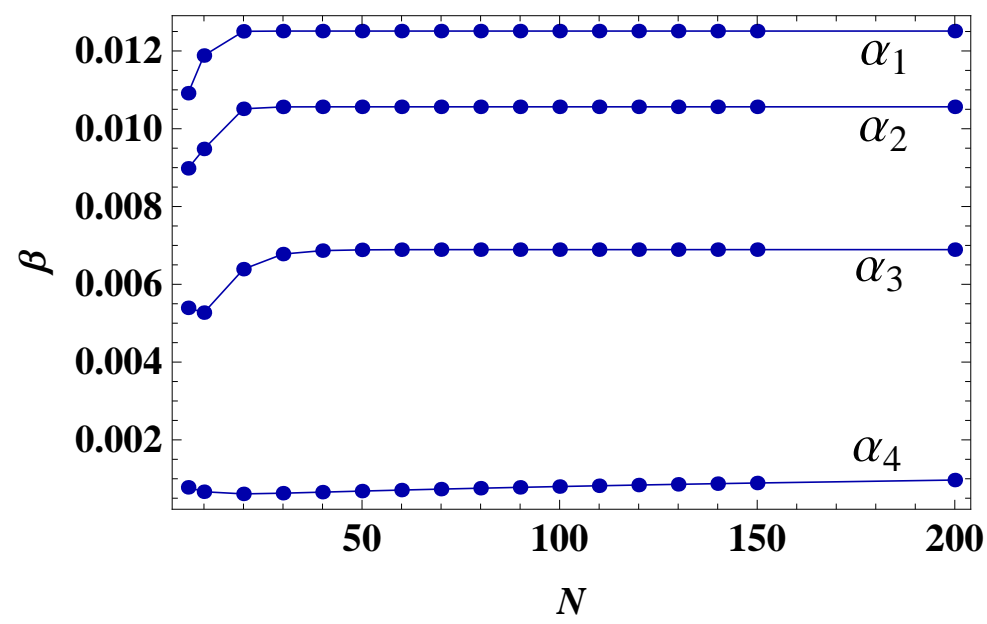

FIG. 10: The function $\beta(N)$.

For $\alpha=\alpha_{1}, \alpha_{2}, \alpha_{3}, \beta$ asymptotically approaches constant values. For the critical case $\alpha=\alpha_{4}$, $\beta$ behaves as

$$
\beta(N) \sim 0.00026 N^{0.32} .
$$


The inequality (41) implies that a large amount of teleported energy requires a large amount of consumption of the ground- state entanglement between the groups $A$ and $B$. In other words, the possible amount of teleported energy is bounded by the amount of entanglement breaking. This relation was previously confirmed for the minimal QET model by one of authors [3]. We have confirmed that a similar relation holds for the QET model with a harmonic chain.

It is noted that our bound (41) with $N=2$ does not quantitatively coincide with the bound of [3] because we treat not a qubit chain as in [3], but a harmonic chain. However, there also exists a qualitative difference between our result with $N=2$ and the result in [3]. Due to noncommutativity between the interaction Hamiltonian and measurement operators, our measurement of $A$ disturbs energy of $B$ directly when $N=2$. Thus the energy gain from $B$ is not purely interpreted as QET output. (However, when $N \geq 4$, the measurement of $A$ does not change energy of $B$; thus, the energy gain from $B$ relies only on QET.) In [3], the measurement of one qubit is a non-demolition measurement for the other qubit. Thus, even in the case of two qubits, the energy gain of [3] is transported by QET.

\section{SUMMARY}

We numerically investigated the protocol of QET for a harmonic chain model. For a bipartite system in the harmonic chain, we applied the QET protocol defined via a POVM measurement and LOCC. The resource for QET in the harmonic chain is the ground-state entanglement between spatially separated two groups. We can extract energy from the system by breaking the entanglement via the POVM measurement and following LOCC.

We confirmed that the amount of extractable energy via the protocol of QET is bounded from above by the amount of the entanglement consumed due to the POVM measurement. This implies that a large amount of teleported energy needs a large amount of consumption of the ground-state entanglement between the two groups in the bipartite system. We also considered the situation that the logarithmic negativity between two groups is zero. In this case, the two groups are separable and there is no quantum correlation between them. Even in such a case, we showed that we can extract energy via the protocol of QED and the amount of energy is correlated with the amount of breaking of the mutual information, which quantifies the total correlation between the two groups. It should be emphasized 
that the above "classical" correlation supporting QET is originally induced from a purely quantum correlation, that is, the ground-state entanglement. If we have no entanglement in the ground state, such "classical" bipartite correlation between $A$ and $B$ does not take place at all. As pointed out above, QET only needs "classical" correlation of $A$ and $B$. This result suggests that QET processes are tolerant of decoherence which destroys the quantum correlation of $A$ and $B$, unlike the usual quantum teleportation.

\section{Acknowledgments}

This research has been partially supported by the Global COE Program of MEXT, Japan, and the Ministry of Education, Science, Sports and Culture, Japan, under Grant No. 22540406.

[1] M. Hotta, "A protocol for quantum energy distribution", Phys. Lett. A 372, (2008) 5671-5676.

[2] M. Hotta, "Quantum Energy Teleportation in Spin Chain Systems", J. Phys. Soc. Jap. 78, (2009) 034001, arXiv:0803.0348.

[3] M. Hotta, "Energy-Entanglement Relation for Quantum Energy Teleportation", Phys. Lett. A 374, (2010) 3416, arXiv:1002.0200 [quant-ph].

[4] M. Hotta, "Quantum energy teleportation with trapped ions", Phys. Rev. A 80, (2009) 042323.

[5] M. Hotta, "Quantum measurement information as a key to energy extraction from local vacuums", Phys. Rev. D 78, (2008) 045006.

[6] M. Hotta, "Quantum energy teleportation with an electromagnetic field: discrete versus continuous variables", J. Phys. A: Math. Theor. 43, (2010) 105305.

[7] M. Hotta, "Controlled Hawking process by quantum energy teleportation", Phys. Rev. D 81, (2010) 044025.

[8] K. Audenaert, J. Eisert, and M. B. Plenio, "Entanglement properties of the harmonic chain", Phys. Rev. A 66, (2002) 042327.

[9] A. Botero and B. Reznik, "Spatial structures and localization of vacuum entanglement in the linear harmonic chain", Phys. Rev. A 70, (2004) 052329.

[10] J. Kofler, V. Vedral, M. S. Kim, and Č. Brukner, "Entanglement between collective operators 
in a linear harmonic chain", Phys. Rev. A 73, (2006) 052107.

[11] Y. Nambu, "Entanglement of Qauntum Fluctuations in the Inflationary Universe", Phys. Rev. $D$ 78, (2008) 044023, arXiv:0805.1471 [gr-qc].

[12] S. Marcovitch, A. Retzker, M. B. Plenio, and B. Reznik, "Critical and noncritical long-range entanglement in Klein-Gordon fields", Phys. Rev. A 80, (2009) 012325.

[13] G. Vidal and R. F. Werner, "Computable measure of entanglement", Phys. Rev. A 65, (2002) 032314 .

[14] M. A. Nielsen and I. L. Chuang, Quantum Computation and Quantum Information (Cambridge Univ. Press, 2000).

[15] A. S. Holevo, M. Sohma, and O. Hirota, "Capacity of quantum Gaussian channels", Phys. Rev. A 59, (1999) 1820-1828.

[16] L. Henderson and V. Vedral, "Classical, quantum and total correlations", J. Phys. A: Math. Gen. 34, (2001) 6899-6905. 